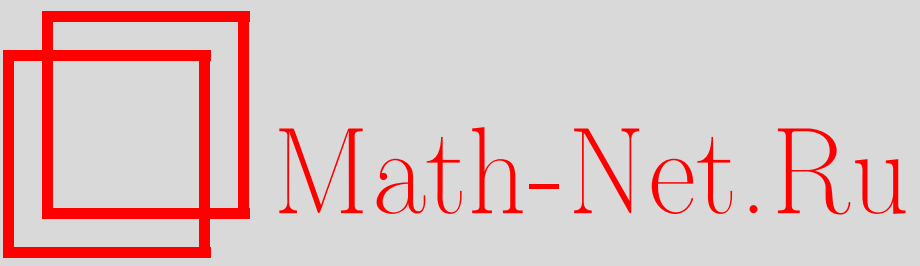

А. Г. Смирнов, М. А. Соловьев, Спектральные свойства виковых степенных рядов свободного поля с индефинитной метрикой, ТМФ, 2000, том 125, номер 1, 57-73

DOI: https://doi.org/10.4213/tmf657

Использование Общероссийского математического портала Math-Net.Ru подразумевает, что вы прочитали и согласны с пользовательским соглашением

http: //www . mathnet.ru/rus/agreement

Параметры загрузки:

IP: 52.87 .193 .239

26 апреля 2023 г., 16:51:25 
ТЕОРЕТИЧЕСКАЯ

И МАТЕМАТИЧЕСКАЯ

ФИЗИКА

Том 125, № 1

октябрь, 2000

(C) 2000 г.

А.Г. Смирнов*, М.А. Соловьев*

\title{
СПЕКТРАЛЬНЫЕ СВОЙСТВА ВИКОВЫХ СТЕПЕННЫХ РЯДОВ СВОБОДНОГО ПОЛЯ С ИНДЕФИНИТНОЙ МЕТРИКОЙ
}

\begin{abstract}
Исследуются свойства бесконечных рядов по виковым степеням свободного поля, двухточечная корреляционная функция которого имеет сингулярное инфракрасное поведение и не удовлетворяет условию положительности. Показано, что при правильном выборе функциональной области определения задаваемые такими рядами поля удовлетворяют всем требованиям псевдовайтмановского формализма. В том случае, когда ряд сходится лишь на аналитических в импульсном представлении пробных функциях, спектральное условие формулируется с помощью введенного ранее понятия несущего конуса аналитического функционала. Для доказательства соблюдения этого условия применяется подходящее обобщение теоремы Пэли-Винера-Шварца.
\end{abstract}

\section{1. ВВЕДЕНИЕ}

В работе [1] установлен критерий, характеризуюший пробные функции, усреднение с которыми обеспечивает сходимость бесконечного ряда по виковым степеням свободного поля в теории с индефинитной метрикой. Здесь мы покажем, что при таком выборе функциональной области определения задаваемые виковыми рядами поля удовлетворяют всем требованиям псевдовайтмановского формализма [2]. Мы также укажем уточнение этого формализма, необходимость которого вытекает из анализа данной задачи. Основная трудность заключается в правильной формулировке спектрального условия в том случае, когда представление группы трансляций псевдоунитарно, а ряд сходится лишь на аналитических в импульсном представлении пробных функциях, что делает невозможной обычную формулировку в терминах носителя спектральной меры или носителя распределения. Важно, что это скорее типичный, чем исключительный случай. Например, такова ситуация с нормальной экспонентой поля дипольного духа [3, 4], входящей в точные решения некоторых калибровочных моделей. Задача соответствуюшего обобшения спектрального условия была поставлена в работе [3], а в [5] предложено воспользоваться для ее решения понятием несушего конуса (квазиносителя) аналитического функционала, успешно применявшимся ранее в нелокальной теории поля. Излагаемые ниже результаты показывают, что этот подход дает точное описание спект-

\footnotetext{
* Физический институт им П. Н. Лебедева РАН, Москва, Россия
} 
ральных свойств виковых рядов и может служить отправной точкой для построения последовательной евклидовой формулировки теории поля с индефинитной метрикой.

В разделе 2 свойства виковых рядов анализируются в рамках традиционного формализма, который применим, если их функциональная область определения содержит функции с компактным носителем как в координатном, так и в импульсном представлениях. В разделе 3 вводятся математические средства, необходимые для анализа общего случая. С этой целью излагаются сведения, касающиеся пространств Гельфанда-Шилова $S_{b}^{a}$, обобщающих введенные также Гельфандом и Шиловым более часто используемые пространства $S_{\beta}^{\alpha}$. Теория пространств $S_{b}^{a}$ была лишь намечена [6], и мы выводим несколько новых теорем, нужных для решения нашей задачи, которые представляют и самостоятельный интерес. В разделе 4 рассматривается преобразование Лапласа аналитических функционалов, определенных на $S_{b}^{a}$. В разделе 5 дана точная формулировка обобшенного спектрального условия и доказано его соблюдение для сумм виковых рядов. Основным средством доказательства служит теорема типа Пэли-Винера-Шварца, установленная в разделе 4 для аналитических функционалов с острым несущим конусом. Раздел 6 посвящен заключительным замечаниям.

\section{2. СВОЙСТВА ВИКОВЫХ РЯДОВ В РАМКАХ ТРАДИЦИОННОГО ФОРМАЛИЗМА}

Пусть $\phi$ - нейтральное скалярное свободное поле, действующее в псевдогильбертовом пространстве состояний $\mathcal{H}$, и пусть $d_{k}-$ коэффициенты ряда по виковым степеням $: \phi^{k}$ : этого поля. Обозначим через $D^{E}$ линейную оболочку вакуума $\Psi_{0}$ и всевозможных векторов вида

$$
\prod_{j=1}^{n}: \phi^{k_{j}}:\left(f_{j}\right) \Psi_{0}
$$

где пробные функции $f_{j}$ пробегают пространство $E$, являющееся подпространством пространства Шварца $S$ и плотное в нем. В работе [1] доказано, что при естественных ограничениях на $d_{k}$ и подходящем выборе $E$ последовательность частичных сумм

$$
\varphi_{N}(f)=\sum_{k \leqslant N} d_{k}: \phi^{k}:(f) \quad(f \in E)
$$

с областью определения $D^{E}$ имеет сильный графф-предел $\varphi(f)$, который является операторнозначной обобщенной функцией над $E$. Более того, сходится любой виков ряд, подчиненный рассматриваемому в том смысле, что его коэффициенты $d_{k}^{\prime}$ удовлетворяют неравенству $\left|d_{k}^{\prime}\right| \leqslant C\left|d_{k}\right|$, и все определяемые такими рядами поля имеют общую плотную и инвариантную область определения $D^{E}(\varphi) \subset \mathcal{H}$. Поскольку операторная реализация строится непосредственно в пространстве состояний исходного свободного поля $\phi$, то часть требований общей теории квантовых полей [2] выполнена для поля $\varphi$ очевидным образом. В частности, по определению сильного графф-предела [7] из взаимной локальности виковых мономов следует, что поле $\varphi$ локально и, более того, взаимно локально с любым полем $\varphi^{\prime}$, задаваемым подчиненным рядом, т.е.

$$
\left[\varphi(f), \varphi^{\prime}\left(f^{\prime}\right)\right] \Psi=0
$$


при любых пробных функциях $f, f^{\prime} \in E$ с пространственноподобно разделенными носителями и при всех $\Psi \in D^{E}(\varphi)$. В пространстве $\mathcal{H}$ исходно имеется псевдоунитарное представление группы Пуанкаре. Реализуюшие его операторы $U(\xi, \Lambda)$ определены на циклической области $D_{0}$ поля $\phi$ и переводят ее в себя. Как показьвают простые примеры, эти операторы не обязательно являются ограниченными, но в силу псевдоунитарности они замыкаемы, и по построению виковых мономов замыкания определены на подпространстве $D^{S}$ и тем более на $D^{E}$. Рассмотрим последовательность $U(\xi, \Lambda) \varphi_{N}(f) U(\xi, \Lambda)^{-1} \Psi_{0}$, где $f \in E$. Согласно закону преобразования виковых мономов, вытекающему из закона преобразования $\phi$, она совпадает с $\varphi_{N}\left(f_{(\xi, \Lambda)}\right) \Psi_{0}$, где $f_{(\xi, \Lambda)}(x)=f\left(\Lambda^{-1}(x-\xi)\right)$. Переходя к пределу $N \rightarrow \infty$ и учитывая замыкаемость оператора $U(\xi, \Lambda)$ и инвариантность вакуума, мы видим, что этот оператор однозначно продолжается на векторы вида $\varphi(f) \Psi_{0}$ и переводит их в $\varphi\left(f_{(\xi, \Lambda)}\right) \Psi_{0}$. Аналогично имеем

$$
U(\xi, \Lambda) \varphi(f) \prod_{j=1}^{n} \varphi\left(f_{j}\right) \Psi_{0}=\varphi\left(f_{(\xi, \Lambda)}\right) \prod_{j=1}^{n} \varphi\left(f_{j(\xi, \Lambda)}\right) \Psi_{0}
$$

при пробных функциях из $E$. Как следствие справедливо равенство

$$
U(\xi, \Lambda) \varphi(f) U(\xi, \Lambda)^{-1} \Psi=\varphi\left(f_{(\xi, \Lambda)}\right) \Psi
$$

при любом $\Psi \in D^{E}(\varphi)$, т.е. условие пуанкаре-ковариантности выполнено.

Пусть d - размерность пространства-времени. Одно из естественных требований к пространству $E$ состоит в том, чтобы тензорное произведение $E\left(\mathbb{R}^{\mathrm{d}}\right)^{\otimes n}$ было плотным в $E\left(\mathbb{R}^{n \mathrm{~d}}\right)$. Тогда полилинейная форма $\Psi\left(f_{1}, \ldots, f_{n}\right)=\varphi\left(f_{1}\right) \ldots \varphi\left(f_{n}\right) \Psi_{0}$ однозначно определяет векторную обобшенную функцию на $E\left(\mathbb{R}^{n \mathrm{~d}}\right)$. Действительно, пусть $f \in E\left(\mathbb{R}^{n \mathrm{~d}}\right), f_{\nu} \in E\left(\mathbb{R}^{\mathrm{d}}\right)^{\otimes n}$ и $f_{\nu} \rightarrow f$ при $\nu \rightarrow \infty$. Имеет место равенство

$$
\left\|\Psi\left(f_{\nu}\right)-\Psi\left(f_{\mu}\right)\right\|^{2}=T_{n}\left(\left(\overline{f_{\nu}-f_{\mu}}\right) \otimes\left(f_{\nu}-f_{\mu}\right)\right),
$$

где обобщенная функция $T_{n}$ выражается через двухточечную вайтмановскую функцию поля $\phi$ и ее гильбертову мажоранту (см. [1]). Пространство $E$ в работе [1] выбрано так, что функция $T_{n}$ определена и непрерывна на $E\left(\mathbb{R}^{2 n \mathrm{~d}}\right)$. Поэтому $\left\|\Psi\left(f_{\nu}\right)-\Psi\left(f_{\mu}\right)\right\| \rightarrow 0$ при $\nu, \mu \rightarrow \infty$. Поскольку пространство $\mathcal{H}$ полно, фундаментальная последовательность $\Psi\left(f_{\nu}\right)$ сходится в нем к некоторому вектору, для которого мы используем стандартную запись

$$
\Psi(f)=\int \varphi\left(x_{1}\right) \ldots \varphi\left(x_{n}\right) f\left(x_{1}, \ldots, x_{n}\right) d x_{1} \ldots d x_{n} \Psi_{0}
$$

Определяемое таким образом отображение $E\left(\mathbb{R}^{n \mathrm{~d}}\right) \rightarrow \mathcal{H}$ линейно и непрерывно. Представление $U(\xi, \Lambda)$ удовлетворяет спектральному условию

$$
\operatorname{supp} \int\langle\Phi, U(\xi, I) \Psi\rangle e^{-i p \xi} d \xi \subset \overline{\mathbb{V}}_{+}
$$

где $\mathbb{V}_{+}-$верхний световой конус, а черта означает замыкание. Исходно здесь $\Phi, \Psi \in D_{0}$, но по построению виковых мономов условие (3) сохраняет силу и для векторов из $D^{S}$, 
причем и в этом случае матричный элемент под знаком интеграла остается полиномиально ограниченным по $\xi$. Подчеркнем, однако, что это свойство ограниченности не обязательно соблюдается для любых $\Phi, \Psi$ из области определения замыкания оператора $U(\xi, I)$. Если фурье-преобразованное пространство $\mathcal{F}(E)$ содержит функции с компактным носителем, то из условия (3) можно обычным образом вывести спектральные свойства функционала $\Psi(f)$, определяемого формулой $(2)$. Пусть $\Phi \in D^{E}(\varphi)$. Функция $\langle\Phi, U(\xi, I) \Psi(f)\rangle$ представляет собой свертку пробной функции $f$ с обобщенной функцией класса $E^{\prime}$ (рассматриваемую на плоскости $x_{1}=\cdots=x_{n}=\xi$ ), поэтому ее рост по $\xi$ ограничен индикаторной функцией, задающей пространство $E$. Пусть $f_{1} \in E\left(\mathbb{R}^{\mathrm{d}}\right)$. Тогда

$$
\int\langle\Phi, U(\xi, I) \Psi(f)\rangle f_{1}(\xi) d \xi=\left\langle\Phi, \Psi\left(f_{2}\right)\right\rangle
$$

где

$$
f_{2}\left(x_{1}, \ldots, x_{n}\right)=\int f\left(x_{1}-\xi, \ldots, x_{n}-\xi\right) f_{1}(\xi) d \xi
$$

Преобразование Фурье переводит $f_{2}$ в

$$
\hat{f}_{2}=\int e^{i p x} f_{2}(x) d x=\hat{f} \hat{f}_{1}\left(p_{1}+\cdots+p_{n}\right) .
$$

Согласно (3) интеграл (4) обращается в нуль, если supp $\hat{f}_{1} \cap \overline{\mathbb{V}}_{+}=\varnothing$. С другой стороны, $\Psi(f) \stackrel{\text { def }}{=} \widehat{\Psi}(\check{f})$, где

$$
\check{f}(p)=(2 \pi)^{-n \mathrm{~d}} \int e^{-i p x} f(x) d x .
$$

Значит, носитель $\widehat{\Psi}$ по переменной $p_{1}+\cdots+p_{n}$ содержится в $\overline{\mathbb{V}}_{-}$. То же самое верно относительно переменной $p_{m}+\cdots+p_{n}, m \leqslant n$, т.е. в импульсном пространстве функционал (2) сосредоточен в конусе

$$
K_{n-}=\left\{p \in \mathbb{R}^{n \mathrm{~d}}: p_{m}+\cdots+p_{n} \in \overline{\mathbb{V}}_{-} \quad \forall m=1, \ldots, n\right\}
$$

В частности, в этом конусе содержится носитель фурье-образа $n$-точечной вайтмановской функции поля $\varphi$, что ясно и из ее явного представления через двухточечную функцию исходного поля $\phi$. Если $\mathcal{F}(E)$ состоит из аналитических функций, изложенный вывод теряет силу и для описания спектральных свойств требуются другие средства функционального анализа.

\section{3. ПРОСТРАНСТВА ГЕЛЬФАНДА-ШИЛОВА-ГУРЕВИЧА}

В работе [1] показано, что в качестве функциональной области определения $E$ викова ряда $\sum d_{k}: \phi^{k}:(x)$ можно взять подходяшее пространство $S_{a}^{b}$. Эти пространства особенно удобны с точки зрения преобразования Фурье, которое просто меняет индексы $a, b$ местами. Задаюшие индексы представляют собой последовательности положительных чисел, подчиненные условиям регулярности

$$
a_{k+l} \leqslant C_{1} h_{1}^{k+l} a_{k} a_{l}, \quad b_{k+l} \leqslant C_{2} h_{2}^{k+l} b_{k} b_{l},
$$


где $C_{1,2}$ и $h_{1,2}$ - константы ${ }^{1)}$. Пространство $S_{b}^{a}=\mathcal{F}\left(S_{a}^{b}\right)$, которое в контексте нашей задачи есть пространство пробных функций в импульсном представлении, состоит из гладких функций, удовлетворяюших ограничениям

$$
\left|p^{\lambda} \partial^{\kappa} g(p)\right| \leqslant C A^{|\kappa|} B^{|\lambda|} a_{|\kappa|} b_{|\lambda|},
$$

где константы $A, B, C$ зависят от $g$, а $\kappa, \lambda$ - мультииндексы, норма которых определяется как сумма компонент. Ограничения (7) можно переписать следуюшим образом:

$$
b\left(\frac{|p|}{B}\right)\left|\partial^{\kappa} g(p)\right| \leqslant C A^{|\kappa|} a_{|\kappa|},
$$

где $b(s)=\sup _{l \in \mathbb{N}}\left(s^{l} / b_{l}\right),|p|=\max _{j}\left|p_{j}\right|$, через $\mathbb{N}$ обозначено множество неотрицательных целых чисел. Функция $b(s)$ называется индикаторной. Если $a_{k}^{1 / k}=O(k)$, то элементы $S_{b}^{a}$ аналитичны, и лишш на таких пробных функциях, как правило, сходятся виковы ряды в теории с индефинитной метрикой, т.е. матричные элементы определяемых ими полей в импульсном представлении являются аналитическими функционалами. Выделенную роль играют пространства, задаваемые последовательностью $a_{k}=k$ !, которые принято обозначать $S_{b}^{1}$. Элементы сопряженного пространства $S_{b}^{\prime 1}$ можно интерпретировать как гиперфункции, растушие на бесконечности не быстрее чем $C_{\epsilon} b(\epsilon|p|)$, где $\epsilon$ сколь угодно мало. Известно, что гиперфункции - это наиболее широкий класс обобщенных функций, для которого корректно определено понятие носителя. В работах $[8,9]$ показано, что часть их локальных свойств наследуют аналитические функционалы класса $S_{b}^{\prime a}$, где $a_{k}=k^{\alpha k}, b_{l}=l^{\beta l}$ и числа $\alpha, \beta$ удовлетворяют условиям $0 \leqslant \alpha<1$, $\beta>1$. Здесь мы распространим развитую в работах $[8,9]$ теорию на более широкий класс функционалов.

ОПРЕДЕЛЕНИЕ 1 . Пусть $\alpha(s)$ и $\beta(s)$ - неотрицательные, непрерывные и монотонно неограниченно возрастающие функции на полуоси $s \geqslant 0$, причем $\alpha(s)$ вьпукла и дифференцируема при $s>0$, а $\beta(s)$ выпукла относительно $\ln s$ и удовлетворяет условию

$$
2 \beta(s) \leqslant \beta(h s)
$$

с некоторой константой $h>1$. Обозначим через $\mathcal{E}_{\beta}^{\alpha}$ пространство, состоящее из целых аналитических функций на $\mathbb{C}^{n}$ таких, что

$$
|g(p+i q)| \leqslant C \exp \left\{\alpha(A|q|)-\beta\left(\frac{|p|}{B}\right)\right\}
$$

где постоянные $A, B, C$ зависят от $g$.

Мы будем использовать обозначение $\alpha_{1} \prec \alpha$, если существуют константы $C, H$ такие, что $\alpha_{1}(s) \leqslant C+\alpha(H s)$.

\footnotetext{
1) Условия (6) обеспечивают выполнимость в $S_{a}^{b}$ операций, требуемых для удобного исчисления. Для вывода результатов работы [1] оказалось достаточным использовать более слабые условия $a_{k+1} \leqslant C_{1} h_{1}^{k} a_{k}, b_{k+1} \leqslant C_{2} h_{2}^{k} b_{k}$.
} 
ТеОрема 1. Пусть $\beta \prec \alpha$. Тогда пространство $\mathcal{E}_{\beta}^{\alpha}$ совпадает с пространством $S_{b}^{a}\left(\mathbb{R}^{n}\right)$, задаваемым последовательностями

$$
a_{k}=\sup _{r \geqslant 0} r^{k} e^{-\alpha_{*}(r)}, \quad b_{l}=\sup _{s \geqslant 0} s^{l} e^{-\beta(s)},
$$

əде $\alpha_{*}(r)=\sup _{s>0}(r s-\alpha(s))$.

Функция $\alpha_{*}$ называется монотонно сопряженной к выпуклой функции $\alpha$ (см. [10]). Условия (6) выполнены для последовательностей (11) ввиду (9) и неравенства $2 \alpha_{*}(s) \leqslant$ $\alpha_{*}(2 s)$, которое при нормировке $\alpha(0)=0$ вытекает из выпуклости $\alpha_{*}$. Для доказательства теоремы 1 нам понадобятся три леммы.

ЛЕмма 1. Для любой функиии $\alpha(s)$, удовлетворяющей условиям определения 1 , при всех $k \geqslant 0$ имеет место равенство

$$
\sup _{r \geqslant 0} r^{k} e^{-\alpha_{*}(r)}=\left(\frac{k}{e}\right)^{k} \inf _{s>0} s^{-k} e^{\alpha(s)}
$$

ДокАЗАТЕЛЬСтво. Подставим $e^{-\alpha_{*}(r)}=\inf _{s>0} e^{\alpha(s)-r s}$ в левую часть равенства (12) и поменяем порядок вычисления верхней и нижней граней. Поскольку $\sup _{r} \inf _{s} G(r, s) \leqslant \inf _{s} \sup _{r} G(r, s)$ для любой функции $G$, то надо убедиться лишь в том, что в нашем случае соблюдается и обратное неравенство, т.е. (по стандартной терминологии [10]) в том, что у функции $G(r, s)=r^{k} e^{\alpha(s)-r s}$ сушествует седловое значение. Справедливость равенства (12) при $k=0$ очевидна, и мы будем считать $k>0$. Напомним, что дифференцируемая выпуклая функция является непрерывно дифференцируемой. Точка $s_{k}$, в которой достигается $\inf _{s>0} e^{\alpha(s)-r s}$, определяется уравнением $\alpha^{\prime}(s)=k / s$, решение которого единственно, так как функция $\alpha^{\prime}$ неотрицательна, монотонна и не равна нулю тождественно. Положим $r_{k}=\alpha^{\prime}\left(s_{k}\right)$. Имеем

$$
\sup _{r \geqslant 0} \inf _{s>0} r^{k} e^{\alpha(s)-r s} \geqslant \inf _{s>0} r_{k}^{k} e^{\alpha(s)-r_{k} s}=\left(\frac{k}{e s_{k}}\right)^{k} e^{\alpha\left(s_{k}\right)}
$$

поскольку функция $\alpha(s)-r_{k} s$ выпукла и для нее любая стационарная точка является точкой абсолютного минимума. Тем самым лемма доказана.

ЛЕмма 2. Для всякой функиии $\beta(s)$, удовлетворяющей условиям определения 1 , при любом $\epsilon>0$ найдется константа $C_{\epsilon}$ такая, что

$$
\beta(s)+\ln s \leqslant C_{\epsilon}+\beta((1+\epsilon) s) .
$$


ДокАЗАТЕльСТво. Для любой неубывающей функции $\beta$, выпуклой относительно $\ln s$ и не сводяшейся к константе, найдется число $c$ такое, что при достаточно больших $s$

$$
\beta(s) \geqslant c \ln s .
$$

Если к тому же соблюдается условие (9), то неравенство (15) верно при сколь угодно большом $c$. Вьпуклость относительно $\ln s$ влечет

$$
\beta(h s) \leqslant \tau \beta\left(h^{1 / \tau} s\right)+(1-\tau) \beta(s)
$$

при $0 \leqslant \tau \leqslant 1$. Положим $\tau=N /(N+1)$ и выберем $N$ так, чтобы $h^{1 / N}<1+\epsilon$. Тогда

$$
\beta(h s)-\beta(s) \leqslant N[\beta((1+\epsilon) h s)-\beta(h s)] .
$$

Здесь $\beta(h s)-\beta(s) \geqslant N \ln s-C_{N}$ ввиду условия (9) и неравенства (15) при $c=N$. Переобозначив $h s \rightarrow s$, получаем (14).

ЛЕмма 3. Пусть функиия $\beta(s)$ удовлетворяет условиям определения 1 , последовательность $b_{l}$ определяется соотношениями (11) $и b(s)=\sup _{l \in \mathbb{N}}\left(s^{l} / b_{l}\right)$. Тогда

$$
b(s) \leqslant e^{\beta(s)} \leqslant C_{\epsilon}^{\prime} b((1+\epsilon) s)
$$

при любом $\epsilon>0$.

ДокАЗАТЕЛЬство. Нетривиально лишш правое неравенство. Будем временно считать $l$ любым действительным числом, не обязательно натуральным, и заметим, что при $l>\beta(e)$ функция $b_{l}$ является неубывающей, поскольку в этом случае верхняя грань в ее определении (11) достигается при $s>1$. Функция $\ln b_{l}$ сопряжена функции $\beta\left(e^{t}\right)$ и конечна при $l \geqslant 0$. Операция сопряжения инволютивна на выпуклых замкнутых функциях (см. [10]), поэтому $\sup _{l \geqslant 0}\left(s^{l} / b_{l}\right)=e^{\beta(s)}$. Если $s$ достаточно велико, то супремум здесь достигается при больших $l$, ибо $\beta(s)$ растет быстрее, чем $c \ln s$ с любым $c$. Пусть $[l]-$ целая часть числа $l$. Тогда

$$
b(s) \geqslant \frac{s^{[l]}}{b_{[l]}} \geqslant \frac{s^{l-1}}{b_{l}}=e^{\beta(s)-\ln s},
$$

и применение неравенства (14) завершает доказательство.

ДОКАЗАТЕЛЬСТВО ТЕОРЕМЫ 1. Пусть $g \in \mathcal{E}_{\beta}^{\alpha}$. При естественной топологизации $\mathcal{E}_{\beta}^{\alpha}$ наименьшая константа $C$, при которой верно (10), есть норма $\|g\|_{A, B}$. Обозначим поликруг $\left\{\zeta \in \mathbb{C}^{n}:|\zeta|=\max _{j}\left|\zeta_{j}\right| \leqslant s\right\}$ через $\mathcal{D}(s)$, а его остов- через $\partial_{0} \mathcal{D}(s)$. Применяя формулу Коши, используя произвол в выборе $s$ и учитывая неравенство $\beta(|p| / 2) \leqslant \beta(\mid \zeta+$ $p \mid)+\beta(|\zeta|)$, вытекающее из монотонности и неотрицательности функции $\beta$, получаем

$$
\begin{aligned}
\left|p^{\lambda} \partial^{\kappa} g(p)\right| & =\left|p^{\lambda}\right| \frac{\kappa !}{(2 \pi)^{n}}\left|\int_{\partial_{0} \mathcal{D}(s)} \frac{g(\zeta+p)}{\zeta^{\kappa+I}} d \zeta\right| \leqslant \\
& \leqslant\|g\|_{A, B}|p|^{|\lambda|} e^{-\beta(|p| / 2 B)}|\kappa| ! \inf _{s>0} s^{-|\kappa|} e^{\alpha(A s)+\beta(s / B)} \leqslant \\
& \leqslant C_{\epsilon}\|g\|_{A, B}\left(2\left(A+\frac{H}{B}+\epsilon\right)\right)^{|\kappa|}(2 B)^{|\lambda|} a_{|\kappa|} b_{|\lambda|},
\end{aligned}
$$


где для получения последнего неравенства мы воспользовались формулой Стирлинга, леммой 1 , условием $\beta \prec \alpha$ и неравенством $\alpha\left(s_{1}\right)+\alpha\left(s_{2}\right) \leqslant \alpha\left(2\left(s_{1}+s_{2}\right)\right)$. Таким образом, пространство $\mathcal{E}_{\beta}^{\alpha}$ непрерывно вложено в $S_{b}^{a}$.

Обратно, пусть $g \in S_{b}^{a}$. Нормы этого пространства будем отмечать штрихом. Используя формулу Тейлора, ограничение (8) и неравенство $|\kappa| ! \leqslant n^{|\kappa|} \kappa !$, получаем следующую оценку для аналитического продолжения функции $g$ в $\mathbb{C}^{n}$ :

$$
|g(p+i q)| \leqslant \sum_{\kappa \in \mathbb{N}^{n}} \frac{\left|q^{\kappa}\right|}{\kappa !}\left|\partial^{\kappa} g(p)\right| \leqslant C_{\epsilon}\|g\|_{A, B}^{\prime} \frac{1}{b(|p| / B)} \sup _{k \in \mathbb{N}}(n(A+\epsilon)|q|)^{k} \frac{a_{k}}{k !} .
$$

В силу леммы 1 и неравенства $1 / k ! \leqslant e^{k} / k^{k}$ мы имеем $a_{k} / k ! \leqslant e^{\alpha(s)} / s^{k}$ при любом $s$. Поэтому супремум по $k$ мажорируется функцией $e^{\alpha(n(A+\epsilon)|q|)}$. Для завершения доказательства остается воспользоваться леммой 3.

ЗАмЕчанИЕ. Хорошо известно, что пространство $S_{b}^{1}$ допускает аналогичное переопределение. Оно может быть задано разрывной выпуклой функцией $\alpha(s)$, равной нулю при $0 \leqslant s \leqslant 1$ и $+\infty$ при $s>1$, и функцией $\beta(s)=\ln b(s)$. Соответственно его элементы аналитичны в областях вида $|y|<1 / A$.

Подкласс пространств типа $S$, характеризуемый теоремой 1 , мы будем называть пространствами Гельфанда-Шилова-Гуревича, поскольку в ряде отношений они близки пространствам типа $W$, рассматривавшимся впервые Гуревичем. Дальнейшее построение теории происходит по той же схеме, что и в работах $[8,9]$, и вместо изложения полных доказательств мы прокомментируем их основные пункты. Ключевым является использование наряду с пространствами $\mathcal{E}_{\beta}^{\alpha}$ родственных пространств над конусами.

ОПРЕДЕЛЕниЕ 2 . Пусть $U$ - открытый конус в $\mathbb{R}^{n}$, а функции $\alpha$ и $\beta$ удовлетворяют условиям определения 1 . Пространство $\mathcal{E}_{\beta}^{\alpha}(U)$ состоит из целых функций на $\mathbb{C}^{n}$ со свойством ограниченности

$$
|g(p+i q)| \leqslant C \exp \left\{\alpha(A|q|)+\alpha \circ \delta_{U}(A p)-\beta\left(\frac{|p|}{B}\right)\right\},
$$

где $\delta_{U}(p)$ - расстояние от точки $p$ до конуса $U$, константы $A, B, C$ зависят от $g$.

ТЕОрема 2. При наделении топологией индуктивного предела относительно семейства банаховых пространств $\mathcal{E}_{\beta, B}^{\alpha, A}(U)$ чельх функиий с нормами

$$
\|g\|_{U, A, B}=\sup _{p, q}|g(p+i q)| \exp \left\{-\alpha(A|q|)-\alpha \circ \delta_{U}(A p)+\beta\left(\frac{|p|}{B}\right)\right\}
$$

пространство $\mathcal{E}_{\beta}^{\alpha}(U)$ становится пространством $\mathrm{DFS}^{2)}$ и, следовательно, является полным отделимым, бочечным , рефлексивным, сеперабельным и монтелевским.

Напомним, что пространства DFS есть пределы инъективных компактных последовательностей локально-вьпуклых пространств. Использование теоремы Монтеля и диагонального процесса позволяет выделить из любой последовательности, содержашейся

\footnotetext{
2) DFS - стандартное обозначение для класса топологических векторных пространств, дуальных пространствам Фреше-Шварца (FS).
} 
в единичном шаре $\mathcal{E}_{\beta, B}^{\alpha, A}(U)$, подпоследовательность, равномерно сходящуюся на компактах в $\mathbb{C}^{n}$ к некоторому элементу этого пространства. В более широком пространстве $\mathcal{E}_{\beta, B^{\prime}}^{\alpha, A^{\prime}}(U), A^{\prime}>A, B^{\prime}>B$, эта сходимость соблюдается по норме, поскольку

$$
\lim _{|q| \rightarrow \infty} e^{\alpha(A|q|)-\alpha\left(A^{\prime}|q|\right)}=0, \quad \lim _{|p| \rightarrow \infty} e^{-\beta(|p| / B)+\beta\left(|p| / B^{\prime}\right)}=0
$$

в силу леммы 2. Таким образом, каноническое отображение $\mathcal{E}_{\beta, B}^{\alpha, A}(U) \rightarrow \mathcal{E}_{\beta, B^{\prime}}^{\alpha, A^{\prime}}(U)$ компактно, что и доказывает теорему 2. По той же причине пространство $\mathcal{E}_{\beta, B+}^{\alpha, A+}(U)=$ $\bigcap_{A^{\prime}>A, B^{\prime}>B} \mathcal{E}_{\beta, B^{\prime}}^{\alpha, A^{\prime}}(U)$ есть пространство FS, что существенно при выводе следующего результата.

ТЕОрема 3. Для любой пары открытых конусов $U_{1}, U_{2} \subset \mathbb{R}^{n}$ справедливо равенство

$$
\mathcal{E}_{\beta}^{\alpha}\left(U_{1}\right) \widehat{\otimes}_{i} \mathcal{E}_{\beta}^{\alpha}\left(U_{2}\right)=\mathcal{E}_{\beta}^{\alpha}\left(U_{1} \times U_{2}\right),
$$

где индекс $і$ указывает на то, что тензорное произведение снабжено индуктивной топологией, а иляпка означает пополнение.

Доказательство такое же, как и доказательство теоремы 3 в работе [9]. Согласно теореме Карлемана-Островского об условиях квазианалитичности пространство $\mathcal{F}\left(\mathcal{E}_{\beta}^{\alpha}\right)$ содержит функции с компактным носителем в том и только том случае, если

$$
\int_{1}^{\infty} \frac{\beta(s)}{s^{2}} d s<\infty
$$

Мы считаем это условие выполненным, поскольку развиваемый формализм нацелен на применение к локальной теории поля.

ТЕОремА 4. При условии (20) пространство $\mathcal{E}_{\beta}^{\alpha}$ плотно в каждом пространстве $\mathcal{E}_{\beta}^{\alpha}(U)$, где $U$ - открытый конус.

Эта теорема доказывается так же, как ее частный случай в работе [9]. Значительно более простое доказательство возможно при дополнительном предположении нетривиальности пространства $\mathcal{E}_{\alpha}^{\alpha}$, что достаточно для большинства интересующих нас приложений. Именно, взяв функцию $e_{0} \in \mathcal{E}_{\alpha}^{\alpha}$ со свойством $\int e_{0}(p) d p=1$, любой элемент $g \in \mathcal{E}_{\beta}^{\alpha}(U)$ можно аппроксимировать функциями $g_{\nu} \in \mathcal{E}_{\alpha}^{\alpha}$, полагая $g_{\nu}=e_{\nu} g$, где $e_{\nu}-$ последовательность римановых сумм для интеграла $\int e_{0}(p-\eta) d \eta$, или более явно

$$
e_{\nu}(p)=\sum_{\kappa \in \mathbb{Z}^{n},|\kappa|<\nu^{2}} e_{0}\left(p-\frac{\kappa}{\nu}\right) \nu^{-n} .
$$

Мы говорим, что замкнутый конус $K \subset \mathbb{R}^{n}$ является несущим конусом функционала $u \in \mathcal{E}_{\beta}^{\prime \alpha}$, если $u$ допускает непрерывное продолжение на каждое пространство $\mathcal{E}_{\beta}^{\alpha}(U)$, где $U \supset K \backslash\{0\}$, иными словами, если $u$ имеет непрерывное продолжение на объединение

$$
\mathcal{E}_{\beta}^{\alpha}(K)=\bigcup_{U \supset K \backslash\{0\}} \mathcal{E}_{\beta}^{\alpha}(U),
$$

наделенное топологией индуктивного предела.

3 Теоретическая и математическая физика, т. 125, № 1, 2000 г. 
Теорема 5. Любой функиионал $u \in \mathcal{E}_{\beta}^{\prime \alpha}$ с несущим конусом $K_{1} \cup K_{2}$ допускает разложсение вида $u=u_{1}+u_{2}$, әде $u_{j} \in \mathcal{E}_{\beta}^{\prime \alpha}\left(K_{j}\right), j=1,2$.

ТЕОРема 6. Если каждый из конусов $K_{1}, K_{2}$ является несущим для функиионала $и \in \mathcal{E}_{\beta}^{\prime \alpha}$, то несущим будет и конус $K_{1} \cap K_{2}$.

Как следствие сушествует наименьший замкнутый конус $K$ такой, что $u \in \mathcal{E}_{\beta}^{\prime \alpha}(K)$. Он может быть назван квазиносителем функционала $u$.

Пространства $\mathcal{E}_{\beta}^{\alpha}(K)$ наследуют топологические свойства, перечисленные в теореме 2. Благодаря этому теоремы 5, 6 равносильны двойственным утверждениям для пробных функций, из которых в доказательстве нуждается лишь утверждение о возможности разложения любой функции $g \in \mathcal{E}_{\beta}^{\alpha}\left(K_{1} \cap K_{2}\right)$ в сумму функций, принадлежащих $\mathcal{E}_{\beta}^{\alpha}\left(K_{1}\right)$ и $\mathcal{E}_{\beta}^{\alpha}\left(K_{2}\right)$. Если $\mathcal{E}_{\alpha}^{\alpha}$ нетривиально, такое разложение не составляет проблемы. Пусть $g \in \mathcal{E}_{\beta, B}^{\alpha, A}(U)$, где $U$ - коническая окрестность пересечения $K_{1} \cap K_{2}$, и пусть $W$ - его чуть меньшая окрестность такая, что $\bar{W} \backslash\{0\} \subset U$. Поскольку угловое расстояние между замкнутыми конусами $K_{j} \backslash W$ не равно нулю, найдутся их конические окрестности $W_{j}$ и число $\theta>0$ такие, что

$$
|p-\eta| \geqslant \theta|p|, \quad|p-\eta| \geqslant \theta|\eta|
$$

при всех $p \in W_{1}, \quad \eta \in W_{2}$. Возьмем любую функцию $e_{0} \in \mathcal{E}_{\alpha, B_{0}}^{\alpha, A_{0}}$ со свойством $\int e_{0}(p) d p=1$ и положим

$$
e(p)=\int_{W_{2}} e_{0}(p-\eta) d \eta
$$

Используя выпуклость $\alpha$ и неравенства (21), можно проверить, что $e g \in \mathcal{E}_{\beta}^{\alpha}\left(K_{1}\right)$ при условии $2 B_{0}<\theta / A$, поскольку тогда функция $e$ убывает в $W_{1}$ не хуже чем $e^{-\alpha(A|p|)}$, что подавляет рост $g$ в этом конусе. Аналогично имеем $(1-e) g \in \mathcal{E}_{\beta}^{\alpha}\left(K_{2}\right)$ при $2 B_{0}<$ $\theta^{\prime} / A$, где $\theta^{\prime}$ - угловое расстояние от $K_{2} \backslash W$ до дополнения $W_{2}$. Если $\mathcal{E}_{\alpha}^{\alpha}$ тривиально, то для вьвода требуемого разложения можно применить стандартный способ решения проблем Кузена с помощью $L^{2}$-оценок Хермандера (см. [8]).

\section{4. ПРЕОБРАЗОВАНИЕ ФУРЬЕ-ЛАПЛАСА В $\mathcal{E}_{\beta}^{\prime \alpha}$}

Перейдем к распространению на функционалы класса $\mathcal{E}_{\beta}^{\prime \alpha}$ теории преобразования Лапласа. Условие неквазианалитичности (20) влечет неравенство $\beta(s)<C_{\epsilon}+\epsilon s$, где $\epsilon$ сколь угодно мало. Поэтому на полуоси $t>0$ определена функция

$$
\beta^{*}(t)=\inf _{s>0}(s t-\beta(s))
$$

Отметим, что она неположительна, вогнута, монотонна и стремится к $-\infty$ при $t \rightarrow 0$.

ТЕОРема 7. Пусть $K$ - острый конус, $V$ - внутренность дуального ему конуса $K^{*}=\{y: p y \geqslant 0 \forall p \in K\}$. Предположим, что выполнено условие (20). Тогда 
любой функционал $u \in \mathcal{E}_{\beta}^{\prime \alpha}(K)$ имеет преобразование Лапласа $\mathbf{v}(z)=\left(u, e^{i(\cdot, z)}\right)$, голоморфное в области $T^{V}=\{z=x+i y: y \in V\}$ и удовлетворяющее оченке

$$
|\mathbf{v}(z)| \leqslant C_{\epsilon}\left(V^{\prime}\right) \exp \left\{\alpha_{*}(\epsilon|z|)-\beta^{*}\left(\frac{|y|}{\epsilon}\right)\right\} \quad\left(y \in V^{\prime}\right)
$$

для любого $\epsilon>0$ и всякого конуса $V^{\prime}$, компактного в $V$ (т.е. такого, что $\bar{V}^{\prime} \backslash\{0\} \subset$ $V)$. При $y \rightarrow 0$ внутри фиксированного конуса $V^{\prime}$ функция $\mathbf{v}(x+i y)$ стремится $\kappa$ фурье-образу функционала и по топологии пространства $\mathcal{F}\left(\mathcal{E}_{\beta}^{\prime \alpha}\right)$.

ДокАЗАТЕЛЬСТво. При любых $A, B>0$ и всяком $U \supset K \backslash\{0\}$ имеем

$$
|\mathbf{v}(z)| \leqslant\|u\|_{U, A, B}\left\|e^{i(p+i q) z}\right\|_{U, A, B},
$$

где

$$
\left\|e^{i(p+i q) z}\right\|_{U, A, B}=\sup _{p} \exp \left\{-p y-\alpha \circ \delta_{U}(A p)+\beta\left(\frac{|p|}{B}\right)\right\} \sup _{q} \exp \{-q x-\alpha(A|q|)\} .
$$

Далее норму $|\cdot|$ в $\mathbb{R}^{n}$ удобно считать евклидовой. При $A>1 / \epsilon$ второй супремум в соотношении (24) мажорируется функцией $e^{\alpha_{*}(\epsilon|x|)}$. Конус $U$ и еше один конус $U^{\prime}$ выберем так, чтобы $U^{\prime}$ был компактен в $\operatorname{int} V^{\prime *}$, а $U$ компактен в $U^{\prime}$. Если $p \notin U^{\prime}$, то $\delta_{U}(p)>\theta|p|$ с $\theta>0$, и, следовательно, $\alpha \circ \delta_{U}(A p)>\alpha(A \theta|p|)$. Кроме того, $\beta(|p| / B)<C+|p| / B$, поэтому при $2 A \theta>1 / \epsilon$ супремум по $p \notin U^{\prime}$ не превосходит $C_{\epsilon} e^{\alpha_{*}(\epsilon|y|)}$. Если $p \in U^{\prime}$, то из включения $\bar{U}^{\prime} \backslash\{0\} \subset \operatorname{int} V^{\prime *}$ следует, что $-p y \leqslant-\theta^{\prime}|p||y|$ при всех $y \in V^{\prime}$. Значит, при $B \theta^{\prime}>1 / \epsilon$ супремум по $p \in U^{\prime}$ не превосходит $-\beta^{*}(|y| / \epsilon)$. Тем самым оценка (23) доказана. Аналогичным образом устанавливается, что при любом $z \in T^{V}$ разностные отношения, соответствуюшие частным производным $\partial e^{i(p+i q) z} / \partial z_{j}$, сходятся к ним в топологии $\mathcal{E}_{\beta}^{\alpha}(U)$, и, следовательно, функция $\mathbf{v}(z)$ голоморфна в этой области. В силу теоремы 1 для функции $f \in \mathcal{F}\left(\mathcal{E}_{\beta}^{\alpha}\right)=S_{a}^{b}$ справедлива оценка $|f(x)| \leqslant C / a(|x| / A)$, где $a(r)=\sup _{k}\left(r^{k} / a_{k}\right) \geqslant c_{\varepsilon} e^{\alpha_{*}(r /(1+\varepsilon))}$ согласно лемме 3 , примененной к функции $\alpha_{*}$. Поэтому $f$ интегрируема с функцией $\left(u, e^{i(\cdot, z)}\right)$ при всяком $y \in V^{\prime}$, причем не только для рассматриваемого, но и для любого $u \in \mathcal{E}_{\beta}^{\prime \alpha}(U)$. Следовательно, последовательность римановых сумм для интеграла

$$
\int e^{i z(p+i q)} f(x) d x
$$

слабофундаментальна в $\mathcal{E}_{\beta}^{\alpha}(U)$ и, ввиду монтелевости этого пространства, сходится в нем. Таким образом,

$$
\int \mathbf{v}(x+i y) f(x) d x=\left(u, e^{-(\cdot, y)} g\right),
$$

где

$$
g(\zeta)=\int e^{i \zeta x} f(x) d x \quad(\zeta=p+i q)
$$


Остается показать, что $e^{-\zeta y} g(\zeta) \rightarrow g(\zeta)$ по топологии $\mathcal{E}_{\beta}^{\alpha}(U)$ при $y \rightarrow 0$ внутри $V^{\prime}$. По теореме о конечных прирашениях имеем $\left|e^{-\zeta y}-1\right| \leqslant|\zeta| \cdot|y| \max _{0 \leqslant t \leqslant 1} e^{-p y t}$. Если $g \in \mathcal{E}_{\beta, B_{0}}^{\alpha, A_{0}}$, то при $A>A_{0}, B>B_{0}$ получаем

$$
\left\|\left(e^{-\zeta y}-1\right) g\right\|_{U, A, B} \leqslant C|y| \max _{0 \leqslant t \leqslant 1} \sup _{p} \exp \left\{-p y t-\alpha \circ \delta_{U}(A p)\right\} .
$$

При $p \in U^{\prime}, y \in V^{\prime}$ выражение под знаком экспоненты отрицательно, а при $p \notin U^{\prime}$ оно не превосходит $\alpha_{*}(|y| / A \theta)$. Теорема доказана.

Пусть $V$ - открытый связный конус в $\mathbb{R}^{n}$. Обозначим через $\mathcal{A}_{\alpha_{*}}^{\beta^{*}}(V)$ пространство функций, голоморфных в $T^{V}$ и удовлетворяюших ограничению (23). При естественной топологизации оно является топологической алгеброй по умножению, поскольку из условия (9) следует $2 \beta^{*}(t) \geqslant \beta^{*}(2 t / h)$. Наиболее важной для нас, но и наиболее трудной задачей является обобщение на аналитические функционалы класса $\mathcal{E}_{\beta}^{\prime \alpha}$ теоремы Пэли-Винера-Шварца. Мы сделаем это при дополнительном ограничении на рост элементов $\mathcal{A}_{\alpha_{*}}^{\beta^{*}}(V)$ на бесконечности. Оно весьма слабое и в интересующих нас приложениях выполнено. Функции $\beta, \beta_{1}$ назовем эквивалентными, если $\beta_{1} \prec \beta$ и $\beta \prec \beta_{1}$. Тогда они задают одно и то же пространство.

Теорема 8. Пусть функции $\alpha, \beta$ обладают свойствами, указанными в определении 1, причем сопряженная $\kappa \alpha$ функция $\alpha_{*}$ растет не бистрее экспоненты первого порядка и конечного типа, а $\beta$ удовлетворяет условию (20) и эквивалентна непрерывной вогнутой функции. Тогда преобразование Лапласа устанавливает изоморфизм между пространством $\mathcal{E}_{\beta}^{\prime \alpha}\left(V^{*}\right)$ и алгеброй $\mathcal{A}_{\alpha_{*}}^{\beta^{*}}(V)$ для любого открытого связного конуса $V$.

ДокАЗАТЕЛЬСтво. Конус $V$ лежит внутри $V^{* *}$, поэтому преобразование Лапласа пространства $\mathcal{E}_{\beta}^{\prime \alpha}\left(V^{*}\right)$ содержится в $\mathcal{A}_{\alpha_{*}}^{\beta^{*}}(V)$ по теореме 7 , и задача состоит в доказательстве обратного включения. Пусть, как и прежде, последовательность $a_{k}$ определяется первой из формул (11). Любая функция $\mathbf{v} \in \mathcal{A}_{\alpha_{*}}^{\beta^{*}}(V)$ имеет граничное значение в смысле теории гиперфункций. При указанном ограничении на рост $\alpha_{*}$ оно определено на пространстве $S_{a}^{1}$, которое при этом ограничении нетривиально ${ }^{3)}$, поскольку содержит целую функцию $\prod_{j=1}^{n} e^{-4 \operatorname{ch} z_{j}}$, мажорируемую функцией $e^{-n e^{|x| / n}}$ при $|y|<\pi / 3$. Элементы $S_{a}^{1}$ аналитичны в областях вида $\{z:|y|<1 / B\}$ и удовлетворяют в них ограничению $|f(z)| \leqslant\|f\|_{A, B} e^{-\alpha_{*}(|x| / A)}$. Поэтому формула

$$
(v, f)=\int \mathbf{v}(x+i y) f(x+i y) d x \quad(y \in V, \quad|y|<1 / B),
$$

где интеграл не зависит от $y$ в силу теоремы Коши-Пуанкаре, определяет линейный непрерывный функционал на $S_{a}^{1}$. Обозначим через $u$ (обратный) фурье-образ $v$ и рассмотрим его свертку с пробной функцией $g \in S_{1}^{a}$. Имеет место тождество

$$
(u * g)(p)=(u, g(p-\cdot))=\int \mathbf{v}(x+i y) e^{-i p(x+i y)} f(x+i y) d x,
$$

\footnotetext{
3) Если рост функции $\alpha$ * такой быстрый, что для любого $N$ найдется $r_{N}$ такое, что $\alpha *(r)>e^{N r}$ при $r>r_{N}$, то $S_{a}^{1}$ тривиально. Это следует из теоремы 4 книги [11, гл. 2].
} 
где

$$
f(z)=(2 \pi)^{-n} \int g(-p) e^{-i p z} d p
$$

Преобразование Фурье является взаимно однозначным и взаимно непрерывным отображением $S_{1}^{a}$ на $S_{a}^{1}$, и сушествуют $A^{\prime}>A, B^{\prime}>B$ такие, что $\|f\|_{S_{a, A^{\prime}}^{1, B^{\prime}}} \leqslant C\|g\|_{S_{1, B}^{a, A}}$. Поэтому из (23), (25) следует оценка

$$
|(u * g)(p)| \leqslant C_{\epsilon, A}\|g\|_{A, B} \inf _{0<t<1 / B^{\prime}} e^{|p| t-\beta^{*}(t / \epsilon)} .
$$

Здесь нижняя грань при достаточно больших $s=|p|$ достигается на отрезке $(0, \delta)$, где $\delta$ сколь угодно мало. Действительно, предположим противное, т.е. что при некотором $\delta>0$ и любом $N$ найдутся $s_{N} \geqslant N$ и $t_{N} \in\left[\delta, 1 / B^{\prime}\right)$ такие, что

$$
s_{N} t_{N}-\beta^{*}\left(t_{N}\right)<\inf _{0<t<\delta}\left(s_{N} t-\beta^{*}(t)\right)
$$

где правая часть не превосходит $s_{N} \delta / 2-\beta^{*}(\delta / 2)$, и, следовательно, $N\left(t_{N}-\delta / 2\right)<$ $\beta^{*}\left(t_{N}\right)-\beta^{*}(\delta / 2)$, что ввиду монотонности $\beta^{*}$ влечет абсурдное неравенство $N \delta / 2<$ $\beta^{*}\left(1 / B^{\prime}\right)-\beta^{*}(\delta / 2)$. Поэтому оценка (26) сохраняет силу, если нижнюю грань брать по всей полуоси и увеличить в случае необходимости константу $C_{\epsilon, A}$. Эквивалентность $\beta$ непрерывной вогнутой функции влечет ее эквивалентность $\beta^{* *}$, в чем легко убедиться, если продолжить эту выпуклую функцию на всю ось, полагая ее равной $-\infty$ при $s<0$, и учесть инволютивность операции сопряжения для замкнутых вьпуклых функций [10]. В итоге мы получаем

$$
|(u * g)(p)| \leqslant C_{\epsilon, A}^{\prime}\|g\|_{A, B} e^{\beta(\epsilon|p|)} .
$$

Согласно лемме 3 работы [1] отсюда следует, что функционал $u$ допускает непрерывное продолжение на $\mathcal{E}_{\beta}^{\alpha}$, причем оно единственно. Остается показать, что конус $V^{*}$ является несущим для этого продолжения. В простейшем случае $V=\mathbb{R}^{n}, V^{*}=\{0\}$ это следует из леммы 1. Действительно, функция $\mathbf{v} \in \mathcal{A}_{\alpha_{*}}^{\beta^{*}}\left(\mathbb{R}^{n}\right)$ является целой и растет не быстрее чем $C_{\epsilon} \exp \left\{\alpha_{*}(\epsilon|z|)\right\}$. Оценка коэффициентов $c_{\kappa}$ представляюшего ее степенного ряда по формуле Коши дает $\left|c_{\kappa}\right| \leqslant C_{\epsilon} \epsilon^{|\kappa|} / a_{|\kappa|}$. Пробная функция $g \in \mathcal{E}_{\beta}^{\alpha}(\{0\})$ также целая и для нее справедливо

$$
|g(p+i q)| \leqslant\|g\|_{A} e^{\alpha(2 A|p+i q|)}
$$

откуда следует оценка

$$
\left|\partial^{\kappa} g(0)\right| \leqslant\|g\|_{A} A^{\prime|\kappa|} \kappa ! \inf _{s>0} s^{-\kappa} e^{\alpha(s)}
$$

При учете формулы Стирлинга соотношение (12) показывает, что функционал

$$
u=\sum_{\kappa \in \mathbb{N}^{n}} i^{\kappa} c_{\kappa} \partial^{\kappa} \delta
$$

определен и непрерывен на $\mathcal{E}_{\beta}^{\alpha}(\{0\})$. 
Дальнейшее рассуждение повторяет доказательство теоремы 4 из работы [9]. Рассмотрим случай $V=\mathbb{R}_{+}, V^{*}=\overline{\mathbb{R}}_{+}$. Применим теорему 5 и разложим $u$ в сумму функционалов $u_{+}, u_{-}$, несомых полуосями $\overline{\mathbb{R}}_{+}$и $\overline{\mathbb{R}}_{-}$. Совершив преобразование Лапласа, получаем $\mathbf{v}(x+i 0)=\mathbf{v}_{+}(x+i 0)+\mathbf{v}_{-}(x-i 0)$, где $\mathbf{v}_{ \pm} \in \mathcal{A}_{\alpha_{*}}^{\beta^{*}}\left(\mathbb{C}_{ \pm}\right)$. По теореме "об острие клина" существует целая функция, которая является продолжением как $\mathbf{v}-\mathbf{v}_{+}$, так и $\mathbf{v}_{-}$. Она растет не быстрее чем $C_{\epsilon} \exp \left\{\alpha_{*}(\epsilon|z|)\right.$, значит, несушим конусом функционала $u-u_{+}$служит начало координат, что завершает доказательство для $n=1$. В обшем случае можно считать, что первый координатный орт лежит в конусе $V$. Возьмем $g \in \mathcal{E}_{\beta}^{\alpha}\left(\mathbb{R}^{n-1}\right)$, введем обозначение $x^{\prime}=\left(x_{2}, \ldots, x_{n}\right)$ и рассмотрим отображение

$$
g \rightarrow \mathbf{v}_{1}\left(z_{1}\right)=\int \mathbf{v}\left(z_{1}, x^{\prime}\right) \check{g}\left(x^{\prime}\right) d x^{\prime}
$$

пространства $\mathcal{E}_{\beta}^{\alpha}\left(\mathbb{R}^{n-1}\right)$ в $\mathcal{A}_{\alpha_{*}}^{\beta^{*}}\left(\mathbb{C}_{+}\right)$. Легко видеть, что оно непрерывно. Пусть $u_{1} \in$ $\mathcal{E}_{\beta}^{\prime \alpha}\left(\overline{\mathbb{R}}_{+}\right)=\mathcal{E}_{\beta}^{\prime \alpha}\left(\mathbb{R}_{+}\right)$- тот функционал, который преобразование Лапласа переводит в $\mathbf{v}_{1}$. Соответствие $\mathbf{v}_{1} \rightarrow u_{1}$ также непрерывно по теореме об открытом отображении [7, теорема V.6]. Тем самым мы получаем билинейный раздельно-непрерывный функционал на $\mathcal{E}_{\beta}^{\alpha}\left(\mathbb{R}_{+}\right) \times \mathcal{E}_{\beta}^{\alpha}\left(\mathbb{R}^{n-1}\right)$, который по теореме 3 определяет линейный непрерывный функционал на $\mathcal{E}_{\beta}^{\alpha}\left(H_{1}\right)$, где $H_{1}$ - полупространство $x_{1}>0$. Его ограничение на $\mathcal{E}_{\beta}^{\alpha}(\mathbb{R}) \otimes \mathcal{E}_{\beta}^{\alpha}\left(\mathbb{R}^{n-1}\right)$ совпадает с ограничением функционала $u$, и мы заключаем, что $\bar{H}_{1}$ является несущим конусом для $u$. То же самое верно для любого из полупространств $\{p: p y \geqslant 0\}$ с $y \in V$, пересечение которых есть конус $V^{*}$, являющийся, следовательно, также несушим для функционала $u$ по теореме 6 , что завершает доказательство.

Полезно иметь еще теорему, характеризующую спектральные функции в том случае, когда свойства регулярности роста голоморфных функций вблизи вешественной границы области аналитичности заранее не известны. Нам будет удобна следующая формулировка.

ТЕОРЕма 9. Пусть функиия $\mathbf{v}$ голоморфна в области $T^{V}$, причем для всякого подконуса $V^{\prime}$, компактного в $V$, и любого $\epsilon>0$ соблюдается неравенство

$$
|\mathbf{v}(z)| \leqslant C_{\epsilon}\left(V^{\prime}\right) \exp \left\{\alpha_{*}(\epsilon|z|)+\gamma_{V^{\prime}}(|y|)\right\} \quad\left(y \in V^{\prime}\right),
$$

где функция $\alpha_{*}$ сопряжена функиии $\alpha$ со свойствами, указанными в определении 1 , и растет не быстрее линейной экспоненты, а $\gamma_{V^{\prime}}$ - неотрицательная, монотонная функиия, неограниченно растущая при уменьшении аргумента. Тогда функиия $\mathbf{v}$ является преобразованием Лапласа аналитического функиионала, принадлежсащего любому пространству $\mathcal{E}_{\beta}^{\prime \alpha}\left(V^{*}\right)$, где функция $\beta$ удовлетворяет условию (20) и такова, что

$$
\left(-\gamma_{V^{\prime}}\right)^{*}(s) \leqslant C_{\epsilon}^{\prime}\left(V^{\prime}\right)+\beta(\epsilon s) .
$$

Действительно, подставляя (28) в (25), мы получаем оценку типа (26) с функцией $\gamma_{V^{\prime}}(t)$ вместо $-\beta^{*}(t / \epsilon)$. Используя монотонность $\gamma_{V^{\prime}}$, в этой оценке можно, как и прежде, перейти к нижней грани по всей полуоси, что в силу соотношения (29) дает прежний результат (27). Оставшаяся часть доказательства такая же, как и в теореме 8 , с учетом неравенства $\gamma_{V^{\prime}}(t) \leqslant C_{\epsilon}^{\prime}\left(V^{\prime}\right)-\beta^{*}(t / \epsilon)$, вытекаюшего из $(29)$. 


\section{5. ОБОБЩЕННОЕ СПЕКТРАЛЬНОЕ УСЛОВИЕ}

Функциональная область определения викова ряда $\sum d_{k}: \phi^{k}:(x)$ установлена в работе [1] исходя из инфракрасного и ультрафиолетового поведения гильбертовой мажоранты вакуумного среднего $w\left(x-x^{\prime}\right)=\left\langle\Psi_{0}, \phi(x) \phi\left(x^{\prime}\right) \Psi_{0}\right\rangle$. Мажоранта является граничным значением функции $\mathbf{w}_{\text {maj }}\left(z, z^{\prime}\right)$, голоморфной в трубчатой области $\left\{\left(z, z^{\prime}\right) \in \mathbb{C}^{2 \mathrm{~d}}\right.$ : $\left.y=\operatorname{Im} z \in \mathbb{V}_{-}, y^{\prime}=\operatorname{Im} z^{\prime} \in \mathbb{V}_{+}\right\}$, и ее поведение можно охарактеризовать оценкой

$$
\left|\mathbf{w}_{\text {maj }}\left(z, z^{\prime}\right)\right| \leqslant C_{0}+C_{1} w_{\mathrm{IR}}\left(|z|+\left|z^{\prime}\right|\right)+C_{2} w_{\mathrm{UV}}\left(|y|+\left|y^{\prime}\right|\right)
$$

где пара $\left(y, y^{\prime}\right)$ пробегает конус, компактный в $\mathbb{V}_{-} \times \mathbb{V}_{+}$, константа $C_{2}$ зависит от этого конуса, а функции $w_{\mathrm{IR}}, w_{\mathrm{UV}}$ монотонны и неотрицательны, причем первая из них растет при увеличении аргумента, а вторая - при его уменьшении. В работе [1] оценка (30) использовалась лишь при $y$ и $y^{\prime}$, принадлежащих оси $y_{0}$, а здесь она нам понадобится в полном объеме.

Пусть ряд $\sum d_{k}: \phi^{k}:(x)$ сходится при усреднении с пробными функциями из пространства $S_{a}^{b}=\mathcal{F}\left(\mathcal{E}_{\beta}^{\alpha}\right)$. Под обобщеннылм спектральным условием мы подразумеваем требование, чтобы замкнутьй конус (5) был несущим конусом векторнозначного аналитического функционала (2) в импульсном представлении. Пусть $\check{f} \in \mathcal{E}_{\beta}^{\alpha}\left(K_{n-}\right), \breve{f}_{\nu} \in \mathcal{E}_{\beta}^{\alpha}$, $\check{f}_{\nu} \rightarrow \check{f}$. Для доказательства соблюдения сфформулированного условия достаточно убедиться, что последовательность $\Psi\left(f_{\nu}\right)=\widehat{\Psi}\left(\check{f}_{\nu}\right)$ фундаментальна. Это так, если острый конус $\left(-K_{n-}\right) \times K_{n-}$ является несущим конусом фурье-образа обобщенной функции $T_{n}$, входящей в правую часть равенства (1). Теорема 9 сводит вывод этого свойства к оценке поведения соответствуюшей аналитической функции $\mathbf{T}_{n}(z)$. При этом надо учесть, что переход из импульсного представления в координатное в квантовой теории поля принято осуществлять с другим знаком в преобразовании Фурье-Лапласа, чем тот, что использовался в предыдущем разделе. В подробной записи $\mathbf{T}_{n}(z)=\sum_{K} D_{K} \mathbf{W}^{K}(z)$, где $z \in \mathbb{C}^{2 n d}, K$ - мультииндекс с компонентами $k_{j m}$, коэффициенты $D_{K}$ выражаются известным образом через $d_{k}$ и

$$
\begin{aligned}
\mathbf{W}^{K}(z)= & \prod_{\substack{1 \leqslant j<m \leqslant n\\
}} \mathbf{w}\left(z_{m}-z_{j}\right)^{k_{j m}} \prod_{n+1 \leqslant j<m \leqslant 2 n} \mathbf{w}\left(z_{j}-z_{m}\right)^{k_{j m}} \times \\
& \times \prod_{\substack{1 \leqslant j \leqslant n \\
n+1 \leqslant m \leqslant 2 n}} \mathbf{w}_{\text {maj }}\left(z_{j}, z_{m}\right)^{k_{j m}} .
\end{aligned}
$$

Обозначим $V_{n-}=\operatorname{int} K_{n-}^{*}, V=V_{n-} \times\left(-V_{n-}\right)$. Тогда

$$
\begin{array}{r}
V=\left\{y \in \mathbb{R}^{2 n \mathrm{~d}}: y_{1} \in \mathbb{V}_{-}, \quad y_{j}-y_{j-1} \in \mathbb{V}_{-}(2 \leqslant j \leqslant n),\right. \\
\left.y_{n+1} \in \mathbb{V}_{+}, \quad y_{j}-y_{j-1} \in \mathbb{V}_{+}(n+2 \leqslant j \leqslant 2 n)\right\} .
\end{array}
$$

Пусть конус $V^{\prime}$ компактен в $V$ и $1 \leqslant j \leqslant n, n+1 \leqslant m \leqslant 2 n$. Если $y$ пробегает $V^{\prime}$, то пара $\left(y_{j}, y_{m}\right)$ пробегает конус, компактный в $\mathbb{V}_{-} \times \mathbb{V}_{+}$, причем найдется $\delta>0$ такое, что $\left|y_{j}\right|+\left|y_{m}\right| \geqslant \delta|y|$. Действительно, обозначим через $\operatorname{pr} V^{\prime}$ проекцию конуса $V^{\prime}$, 
т.е. его пересечение с единичной сферой в $\mathbb{R}^{2 n d}$, и рассмотрим выражение $\left|y_{j}\right|+\left|y_{m}\right|$ как функцию $y$. Она непрерывна, отображает компакт $\operatorname{pr} V^{\prime}$ в компактное подмножество $\mathbb{V}_{-} \times \mathbb{V}_{+}$и достигает на $\operatorname{pr} V^{\prime}$ своей нижней грани, которая не может быть нулем, ибо точки с нулевыми компонентами не принадлежат $V$. Отсюда и следует наше утверждение. Аналогичное рассуждение показывает, что при индексах $1 \leqslant j<m \leqslant n$ и $n+1 \leqslant j<m \leqslant 2 n$ разность $y_{j}-y_{m}$ компонент вектора $y \in V^{\prime}$ пробегает конусы, компактные соответственно в $\mathbb{V}_{+}$и $\mathbb{V}_{-}$, причем $\left|y_{j}-y_{m}\right| \geqslant \delta|y|$ с другой, быть может, но ненулевой константой $\delta$. Используя доказанное в работе [1] неравенство $\left|\mathbf{w}\left(x-x^{\prime}-2 i \eta\right)\right|^{2} \leqslant\left|\mathbf{w}_{\text {maj }}(x-i \eta, x+i \eta)\right|\left|\mathbf{w}_{\text {maj }}\left(x^{\prime}-i \eta, x^{\prime}+i \eta\right)\right|$, верное при всех $\eta \in \mathbb{V}_{+}$, применяя оценку (30) и учитывая монотонность функций $w_{\mathrm{IR}}, w_{\mathrm{UV}}$, получаем

$$
\left|\mathbf{W}^{K}(z)\right| \leqslant 3^{|K|}\left(C_{0}^{|K|}+C_{1}^{|K|} w_{\mathrm{IR}}(2|z|)^{|K|}+C_{2}\left(V^{\prime}\right)^{|K|} w_{\mathrm{UV}}\left(\delta_{V^{\prime}}|y|\right)^{|K|}\right) \quad\left(y \in V^{\prime}\right) .
$$

На коэффициенты рассматриваемых виковых рядов мы накладываем ограничения

$$
d_{k} \geqslant 0, \quad d_{0}=1, \quad \lim _{k \rightarrow \infty}\left(k ! d_{k}^{2}\right)^{1 / k}=0, \quad d_{k} d_{l} \leqslant C H^{k+l} d_{k+l}
$$

( $C, H$ - константы), смысл которых разъяснен в [1]. Третье из ограничений (32) обеспечивает абсолютную сходимость представляющего функцию $\mathbf{T}_{n}(z)$ ряда в области $T^{V}$ и, следовательно, голоморфность в ней этой функции, а четвертое позволяет вывести из оценки (31) неравенство

$$
\left|\mathbf{T}_{n}(z)\right| \leqslant C\left(\sum_{k=0}^{\infty} L^{k} k ! d_{2 k} w_{\mathrm{IR}}(2|z|)^{k}\right)\left(\sum_{k=0}^{\infty} L^{k} k ! d_{2 k} w_{\mathrm{UV}}(\delta|y|)^{k}\right) \quad\left(y \in V^{\prime}\right)
$$

где $L$ и $\delta$ зависят от $V^{\prime}$.

Теперь можно подвести итог нашему рассмотрению.

ТЕОрема 10. Пусть $\phi-$ свободное поле, действующее в псевдогильбертовом пространстве $\mathcal{H}$. При выполнении условий (32) на коэффициенты поле $\varphi(x)=$ $\sum d_{k}: \phi^{k}:(x)$ корректно определено как операторная обобщенная функция на всяком пространстве $S_{a}^{b}$, индикаторные функции которого удовлетворяют неравенствам

$$
\sum_{k} L^{k} k ! d_{2 k} w_{\mathrm{IR}}(r)^{k} \leqslant C_{L, \epsilon} a(\epsilon r), \quad \inf _{t>0} e^{s t} \sum_{k} L^{k} k ! d_{2 k} w_{\mathrm{UV}}(t)^{k} \leqslant C_{L, \epsilon} b(\epsilon s)
$$

при сколь угодно большом $L>0$ и сколь угодно малом $\epsilon>0$, и, кроме того, функщия $\ln a(r)$ растет не бъстрее линейной әкспоненты. Если $S_{b}^{a}$ является пространством Гельфанда-Шилова-Гуревича и функиия $\beta(s)=\ln b(s)$ удовлетворяет условию (20), то для $\varphi(x)$ выполняются все требования псевдовайтмановского формализма, включая обобщенное спектральное условие.

Первое утверждение доказано в работе [1], а второе выводится из неравенства (33) с помошью теоремы 9 , если взять $\gamma_{V^{\prime}}(t)=\ln \sum_{k} L_{V^{\prime}}^{k} k ! d_{2 k} w_{\mathrm{UV}}\left(\delta_{V^{\prime}} t\right)^{k}$. 


\section{6. ЗАКЛЮЧЕНИЕ}

Функциональная область определения виковых рядов свободного поля с индефинитной метрикой была найдена в работе [1] относительно простым способом. Напротив, доказательство соблюдения для предела ряда основных требований обшей теории квантовых полей потребовало довольно глубокого изучения свойств аналитических функционалов. Мы считаем эти усилия оправданными, поскольку они не только позволяют сделать необходимые уточнения псевдовайтмановского формализма, но и дают, по нашему мнению, математическую основу для последовательной евклидовой формулировки квантово-полевых моделей с сингулярным инфракрасным поведением, нарушающим условие положительности. Используемое выше ограничение на рост индикаторной функции $a(r)$ не является сушественным и мотивировано лишь желанием придать доказательствам бо́льшую наглядность и краткость. Стоит отметить, что рассматриваемые виковы ряды всегда можно реализовать на пространствах $S_{0}^{b}$, которым соответствуют пространства $\mathcal{E}_{\beta}^{\alpha}$ с $\alpha(s)=s$. Эти пространства допускают любое инфракрасное поведение, поскольку состоят из функций с компактным носителем в координатном представлении, и соответствуюшая формулировка спектрального условия является наиболее слабой (т.е. наиболее обшей). Однако целью данной работы было точное описание как функциональной области определения виковых рядов, так и их спектральных свойств. Развитая техника позволяет полностью решить еше одну интересную задачу - исследовать нелокальное расширение классов эквивалентности Борхерса, где основная трудность связана с правильным обобщением условия микропричинности и доказательством его соблюдения для нелокальных виковых рядов. Эти результаты будут изложены в отдельной работе.

Благодарности. Авторы признательны за финансовую поддержку, оказанную работе грантами РФФИ-99-02-17916 и INTAS-96-0308.

\section{Список литературы}

[1] А.Г. Смирнов, М. А. Соловьев. ТМФ. 2000. Т. 123. № 3. С. 355.

[2] Н. Н. Боголюбов, А. А. Логунов, А. И. Оксак, И. Т. Тодоров. Общие принципы квантовой теории поля. М.: Наука, 1987.

[3] U. Moschella, F. Strocchi. Lett. Math. Phys. 1992. V. 24. P. 103.

[4] U. Moschella. J. Math. Phys. 1993. V. 34. P. 535.

[5] М. А. Соловьев. ТМФ. 1995. Т. 105. С. 405

[6] И. М. Гельфанд, Г. Е. Шилов. Обобщенные функции. Т. 2. М.: Физматгиз, 1958.

[7] М. Рид, Б. Саймон. Методы современной математической физики. Т. 1. М.: Мир, 1977.

[8] M. A. Soloviev. Lett. Math. Phys. 1995. V. 33. P. 49.

[9] M. A. Soloviev. Commun. Math. Phys. 1997. V. 184. P. 579.

[10] Р. Рокафеллар. Выпуклый анализ. М.: Мир, 1973.

[11] С. Мандельбройт. Теоремы замкнутости и теоремы композиции. М.: ИЛ, 1962.

Поступила в редакцию 30.III.2000 г. 